\title{
Carotid Sinus Syndrome in a Patient with Symptomatic Abdominal Aortic Aneurysm - A Case Report
}

\author{
Ksenija Stevanovic ${ }^{1}$, Ranko Trailovic ${ }^{1}$, Biljana Kukic ${ }^{1}$, Marko Dragas ${ }^{2,3}$, Slobodan Cvetkovic ${ }^{2,3}$ and \\ Lazar Davidovic ${ }^{2,3}$
}

${ }^{1}$ Center for Anesthesiology and Resuscitation, Clinical Center of Serbia, Serbia

${ }^{2}$ Clinic for Vascular and Endovascular Surgery, Clinical Center of Serbia, Serbia

${ }^{3}$ School of Medicine, University of Belgrade, Serbia

*Corresponding author: Ksenija Stevanovic, Center for Anesthesiology and Resuscitation, Clinical Center of Serbia, Pasterova 2, 11000 Belgrade, Serbia, Tel: +381668300966

\begin{abstract}
Carotid sinus syndrome (CSS) is defined as syncope with reproduction of symptoms during 10 s of carotid sinus massage. The etiology of this condition is unknown, patients are usually symptomatic and present with episodes of syncope. Due to the associated marked sinus bradycardia, sinus arrest or severe hypotension, carotid sinus syndrome may be life-threatening. In patients without previous symptoms, CSS must be promptly recognized and treated, otherwise may lead to catastrophic consequences. If symptoms of CSS appear for the first time during urgent symptomatic abdominal aortic aneurysm surgery consequences might be devastating. Herein, we describe a case of successful management of a patient with carotid sinus syndrome who has undergone urgent symptomatic abdominal aortic aneurysm open repair.
\end{abstract}

\section{Keywords}

Carotid sinus syndrome, Carotid sinus massage, Abdominal aortic aneurysm, Open repair

\section{Introduction}

Carotid sinus syndrome (CSS) is characterized by a decrease in heart rate and/or blood pressure in response to carotid sinus massage (CSM). Epidemiological data are lacking and the only fact known for sure is that the prevalence of CSS is extremely low below the age of 50 and increases exponentially with increasing age [1]. Due to the associated marked sinus bradycardia, sinus arrest or severe hypotension this condition may be life-threatening.
Symptomatic abdominal aortic aneurysm (sAAA) requires urgent surgical intervention, due to high possibility of rupture [2]. If symptoms of CSS appear for the first time during urgent symptomatic abdominal aortic aneurysm surgery consequences might be devastating. Herein, we describe a case of successful management of a patient with CSS who has undergone sAAA open repair.

\section{Case Description}

A 57-year-old male was admitted to the Emergency Center, Clinical Center of Serbia, due to continuous and increasing pain in the abdomen, caused by pulsatile palpable mass. Following ultrasound, multislice computed tomography scan revealed infrarenal aortic aneurysm, with a maximum diameter of $70 \mathrm{~mm}$, without signs of thrombosis, dissection or rupture. Visceral and carotid arteries showed no significant hemodynamic and morphological alterations. The patient was urgently transferred to our Institution. On admission he was hemodynamically stabile (BP 160/78 mmHg, HR 94 beats/min). The patient's medical history, besides hypertension was negative for any other chronic diseases and surgical interventions. Upon basic preoperative investigations, the patient was transferred to the operating theatre. Following routine general endotracheal anesthesia induction and central venous catheter placement, during nasogastric tube insertion, severe bradycardia and hypotension occurred, instantly followed by asystole.

Citation: Stevanovic K, Trailovic R, Kukic B, Dragas M, Cvetkovic S, et al. (2018) Carotid Sinus Syndrome in a Patient with Symptomatic Abdominal Aortic Aneurysm - A Case Report. Int J Clin Cardiol 5:130. doi.org/10.23937/2378-2951/1410130

Accepted: November 10, 2018; Published: November 12, 2018

Copyright: (c) 2018 Stevanovic K, et al. This is an open-access article distributed under the terms of the Creative Commons Attribution License, which permits unrestricted use, distribution, and reproduction in any medium, provided the original author and source are credited. 
Urgent therapeutic measures were undertaken: chest compressions were initiated and adrenalin boluses $(1+$ $1 \mathrm{mg}$ ) were administered. Since sinus rhythm was briefly restored and hemodynamic stabilization of the patient was achieved, surgical procedure was resumed. The rest of the intraoperative course was uneventful. Postoperative serial 12-lead electrocardiogram (ECG) and transthoracic echocardiography revealed no alterations. On the $2^{\text {nd }}$ postoperative day another episode of bradycardia with hypotension occurred when patient performed extreme neck rotation. Bilateral CSM revealed positive CSS signs on the left: asystole $>8$ sec along with a drop in systolic blood pressure for $65 \mathrm{mmHg}$, so diagnosis was confirmed. The patient's head was maintained in a neutral position and on the $3^{\text {rd }}$ postoperative day anti-bradycardia dual-chamber, rate-modulated pacemaker (DDDR, St Jude Medical, Zephyr XL DR, model 5826) was implanted. Postoperative course was uncomplicated, so on the $7^{\text {th }}$ postoperative day the patient was discharged in good general medical condition.

According to the Guidelines of the European Society of Cardiology CSS is defined as syncope with reproduction of symptoms during 10s of CSM [3]. Depending on the type of CSM response, there are three CSS subtypes: cardioinhibitory, vasodepressor and the least frequent-mixed form, seen in our patient. Although the etiology is unknown, disturbances in autonomic nervous system are considered to be the main pathophysiological basis of this condition, involving a pathological reflex. Receptors in the carotid sinus are sensitive to stretching of the arterial wall and fire impulses through a branch of the glossopharyngeal nerve-Hering's nerve to the nucleus tractus solitarii in the medulla oblongata. The efferent limb of the reflex is carried via vagal nerve and cervical sympathetic nerves to the heart and vessels, controlling heart rate and vasomotor tone [4]. The abnormal reflex might develop due to malfunction of the baroreceptor and due to degeneration of the medulla $[5,6]$. Some trigger mechanisms have particular tendency to provoke CSS symptoms, and most commonly those include: head and neck movement, tight collars, hyperextension of the neck, constricting neckwear, tumors, surgery or irradiation close to the carotid sinus $[7,8]$. Also, triggering actions such as coughing, sneezing, straining for stool, and lifting of heavy objects, and even difficult tracheal intubation have been described to cause CSS $[9,10]$. In most cases, no obvious precipitating CSS cause can be identified.

That was the case with our patient: first symptoms appeared intraoperatively, during nasogastric (NG) tube placement and neck manipulations during that process. Since such blood pressure and cardiac rhythm disturbances did not repeat, that episode was attributed to vagal nerve stimulation. When the second episode occurred CSS was suspected and confirmed. Although the patient had no prior history of syncope, due to clinical suspicion and timely diagnosis, he received proper treatment. The only question that remains unclear is why CSS symptoms appeared exactly during the perioperative period? A possible explanation might be that hemodynamically challenging surgical intervention as sAAA urgent open repair is, along with anesthesia provoked and worsened hypersensitive carotid sinus abnormalities. The majority of reports in the available literature describe CSS in patients with neck tumors $[7,8]$. Our patient's history was negative for any head and neck tumors, so to the best of our knowledge this is the first paper reporting about CSS in a patient with sAAA. Furthermore, the fact that CSS symptoms became evident for the first time intraopertively gives greater significance to this report.

\section{Conclusion}

Symptoms of CSS may become evident during urgent and hemodynamically challenging surgical interventions. Although rare, CSS might lead to severe bradycardia, marked hypotension or even cardiac arrest, so medical teams (even in surgical settings) involved in the treatment of such a patient should always keep in mind the fact that timely diagnosis and urgent therapeutic measures are necessary, in order to achieve positive outcomes.

\section{Conflict of Interest}

$\mathrm{KS}, \mathrm{RT}, \mathrm{BK}, \mathrm{MD}, \mathrm{SC}$, and LD declare no conflict of interest.

\section{Author's contribution}

KS, RT, BK, MD, SC and LD have equally contributed to the manuscript preparation.

\section{References}

1. Humm AM, Mathias CJ (2006) Unexplained syncope-is screening for carotid sinus hypersensitivity indicated in all patients aged $>40$ years? J Neurol Neurosurg Psychiatry 77: $1267-1270$.

2. Matsushita M, Ikezawa T, Sugimoto M, Idetsu A (2014) Management of symptomatic abdominal aortic aneurysms following emergency computed tomography. Surg Today 44: $620-625$

3. Brignole M, Auricchio A, Baron-Esquivias G, Bordachar P, Boriani G, et al. (2013) 2013 ESC Guidelines on cardiac pacing and cardiac resynchronization therapy: The task force on cardiac pacing and resynchronization therapy of the European Society of Cardiology (ESC). Developed in collaboration with the European Heart Rhythm Association (EHRA). Eur Heart J 34: 2281-2329.

4. De Miranda Félix RH, Medeiros da Fonseca ARB, HCS Silva $B$, Góis AFT (2015) Carotid sinus syndrome in a patient with Hodgkin's lymphoma. IJCRM 2015: 1-4.

5. Healey J, Connolly SJ, Morillo CA (2004) The management of patients with carotid sinus syndrome: Is pacing the answer? Clin Auton Res 14: 80-86.

6. Miller VM, Kenny RA, Slade JY, Oakley AE, Kalaria RN (2008) Medullary autonomic pathology in carotid sinus hypersensitivity. Neuropathol Appl Neurobiol 34: 403-411. 
7. Mehta $N$, Abdelmessih $M$, Smith L, Jacoby $D$, Marieb $M$ (2014) Carotid sinus syndrome as a manifestation of head and neck cancer-case report and literature review. Int J Clin Cardiol 1: 1-5.

8. Truong AT, Sturgis EM, Rozner MA, Truong DT (2013) Recurrent episodes of asystole from carotid sinus hypersensitivity triggered by positioning for head and neck surgery. Head Neck 35: E28-E30.
9. Wenger TL, Dohrmann ML, Strauss HC, Conley MJ, Wechsler AS, et al. (1980) Hypersensitive carotid sinus syndrome manifested as cough syncope. Pacing Clin Electrophysiol 3: 332-339.

10. Plotkin CN, Eckenbrecht PD, Waldo DA (1989) Consecutive cardiac arrests on induction of anesthesia associated with nifedipine-induced carotid sinus hypersensitivity. Anesth Analg 68: 402-405. 